underpinned the development of the 'Talk-to-Me' MOOC which was launched in March 2020. To date this MOOC has enrolled over 45,000 participants from over 150 countries, with the average age of users being 24 years. Collectively, this line of work highlights that MOOCs are an effective means of mental health promotion to young adults.

\section{Burnout and Interventions for Healthcare workers to cope during COVID-19}

Harnek Kailey*

Maidstone and Tunbridge Wells NHS Trust

${ }^{*}$ Corresponding author.

doi: 10.1192/bjo.2021.404

Aims. Healthcare workers are exposed to both physical and mental demands in the hospital environment; recently intensified by overstretching staff and resources during the current COVID-19 pandemic. Despite healthcare workers banding together, physician burnout is more prevalent than ever before due to emotional, physical and mental exhaustion. Firstly, this poster aims to expose the prevalence of burnout among healthcare workers during the COVID-19 pandemic. Secondly, to highlight the interventions and strategies to help minimise burnout among healthcare workers. Method. I will focus on reviewing clinical trials with a particular focus on healthcare workers affected by burnout within the COVID-19 pandemic timeframe. Therefore, narrowing my search to 20 trials within the past 12 months using the following restricted search criteria: 'burnout', 'covid-19' and 'healthcare'. Furthermore, commenting on strategies and interventions to minimise burnout by stretching my criteria to interventions trialled within the last 24 months. This is due to limited data and trial evidence for burnout strategies within the last 12 months of the COVID-19 pandemic.

Result. Burnout is on the rise among healthcare workers across the globe, $47 \%$ of healthcare staff are expressing an element of burnout worldwide. With growing concerns of healthcare staff developing long term mental health implications as a result of work-related stress. At present, one third of frontline staff are experiencing depression and distress; which must be addressed. Reviewing recent trials has highlighted a number of successful strategies for approaching burnout including: app technology, talking therapy, staff support and internet-based resources. App-related technology and web resources have shown to be particularly beneficial among recent trials, with limited participation and engagement for support groups/talking therapy.

Conclusion. A significant rise in physician burnout and distress during COVID-19 has been noted in various trials. Interventions specifically related to burnout within COVID-19 are limited due to a low yield of completed trials; however, a couple of trials have found an improvement in COVID-19 related stress among healthcare workers using app-related technology. Internet based intervention is cheap, widely accessible and a non-judgemental method for seeking help, especially within a profession where burnout is heavily stigmatized.

Motivators and deterrents in choosing a career in psychiatry; making the most of psychiatry school events

Nosheen Kazmi ${ }^{1 \star}$, Emily Lewis ${ }^{2}$, Catarina Cardoso Rodrigues dos Santos $^{3}$ and Sahana Olety ${ }^{4}$

${ }^{1}$ ST4 CAMHS, Pennine Care NHS Foundation Trust; ${ }^{2}$ Locum ST, Cheshire and Wirral Partnership NHS Foundation Trust; ${ }^{3}$ ST4

Forensic Psychiatrist, Greater Manchester Mental Health and
${ }^{4}$ Consultant Child and Adolescent Psychiatrist, UK, Pennine Care NHS Foundation Trust

${ }^{*}$ Corresponding author.

doi: 10.1192/bjo.2021.405

Aims. In response to the Royal College of Psychiatrists' recruitment strategy, a bi-annual Psychiatry School event was set up in the North West of England. The Psychiatry School aims to inspire medical students and foundation doctors to choose a career in Psychiatry with two days of workshops on different subspecialties and various aspects of the career pathway. A previous service evaluation has shown attending the event improves attitudes towards psychiatry.

The aim is to assess whether improving attitudes to psychiatry has been sustained and gain a clearer understanding of the motivators and deterrents in choosing a career in Psychiatry to better inform future events.

Method. An online questionnaire about positive and negative aspects of psychiatry was sent to attendees of the Autumn North West Psychiatry School 2020 before and after the event. Result. The total number of completed questionnaires was 62 .

$53.6 \%$ people were considering applying for core psychiatry training prior to the event and this rose to $85.3 \%$ in the post event questionnaire.

Motivators for a career in psychiatry prior to the event included having a better holistic understanding of patients and wide range of sub-specialities. There was a common theme of interest in research opportunities. Dynamic patient-doctor relationship, exploring issues in depth and treating diverse populations were key motivators.

It is encouraging to note that $100 \%$ responders felt their positive views on psychiatry were validated.

The majority of deterrents were disregarded and attendees felt positive about choosing a career in psychiatry.

Conclusion. Following the event, the only negative view on a career in Psychiatry was the concern about the potential impact on one's own mental health. This is an important issue (highlighted in the Royal College of Psychiatrists Position Statement) that deserves consideration at future events to highlight potential effects on Psychiatrists wellbeing and how these can be avoided or mitigated.

The wide variety of sub-specialities and opportunities for research were key areas that motivated attendees and we will continue to deliver engaging workshops around these themes.

\section{Digital frontiers in international psychiatric} recruitment: the lessons of the Northwest School of Psychiatry careers event November 2020

Nosheen Kazmi ${ }^{1 \star}$, Catarina Rodrigues dos Santos ${ }^{2}$, Emily Lewis ${ }^{3}$ and Sahana Olety ${ }^{4}$

${ }^{1}$ ST4 CAMHS, Pennine Care NHS Foundation Trust; ${ }^{2}$ ST4 Forensic Psychiatrist, Greater Manchester Mental Health; ${ }^{3}$ Specialist Doctor in Psychiatry, Cheshire and Wirral Partnership NHS Foundation Trust and ${ }^{4}$ Foundation Tutor, Pennine Care NHS Foundation Trust, Consultant Child and Adolescent Psychiatrist

${ }^{\star}$ Corresponding author.

doi: 10.1192/bjo.2021.406

Aims. A low level of psychiatric recruitment is a global issue[1]. The RCPsych \& UK Mental Health Trusts jointly run School Events as part of a recruitment strategy. The North West has been running such events for the past years. After our first virtual event, we compare the quality, effectiveness and experience of a 